\title{
Development of an intelligent system of experimental control in the energy sector
}

\author{
Larisa Grigoreva ${ }^{1, *}$, and Vladimir Grigoryev ${ }^{2}$ \\ ${ }^{1}$ Moscow State University of Civil Engineering, 129337, 26 Yaroslavskoe sh., Moscow, Russian \\ Federation \\ ${ }^{2}$ Moscow branch of Centrtomtechenergo «Atomtechenergo» JSC, 115432, 6 Proyektiruyemyy Ave. \\ 4062, Moscow, Russian Federation
}

\begin{abstract}
The article presents a completed project of an experimental control system in the energy sector. An intelligent experimental control system can be used at various types of power plants, including nuclear power units. The developed complex includes design schemes for operational monitoring and registration of rapidly changing neutronphysical and technological parameters of a nuclear power unit at the stages of commissioning of a power unit. Automated process control systems allow the collection and registration of analog parameters with a frequency of not more than 1 second, which is insufficient for correct calculations during neutron-physical experiments and power unit tests. The presented intelligent experimental control system allows the reception and registration of analog signals with a frequency of not more than 100 millisecond. A patented software implementation has been developed that provides unlimited possibilities for realizing customer requirements for operator functionality and interface, as well as the ability to avoid tight binding to specific hardware components.
\end{abstract}

\section{Introduction}

The development of modern society is inextricably linked with the development of the energy sector. The main challenges facing the energy industry are environmental and energy security, energy availability. The structure of primary energy consumption is changing. There is a transition to the widespread use of renewable energy sources (RES). The energy potential of renewable energy is many times higher than the current level of energy consumption, and therefore they can be considered as a possible source of energy production. Serious disadvantages of RES include the low density of energy flows and their inconstancy in time. Significant costs for equipment providing the collection, storage and conversion of energy [1,2]. According to information from the International Renewable Energy Agency (IRENA), it is shown that an increase in the share of renewable energy sources to $34 \%$ is possible by 2030 [3]. According to the forecast of the Institute for Energy Research of the Russian Academy of Sciences and the Center for Energy of the Moscow

${ }^{*}$ Corresponding author: pehel@yandex.ru 
School of Management SKOLKOVO, along with traditional energy sources, RES will play a major role: by 2040 , renewable energy sources can provide $35-50 \%$ of global electricity production and $19-25 \%$ of total energy consumption [4]. Along with renewable energy, nuclear energy provides access to clean, reliable and affordable energy, mitigating the negative effects of climate change. It makes up a significant part of the global energy balance. Nuclear power plants (NPPs) occupy a special place. According to the authors of [4], in the long term until 2040 the growth of world production at nuclear power plants will lag behind the growth rate of electricity consumption. Installed capacity of nuclear power plants will grow by $45-51 \%$ compared to 2015 by 2040 . Studies [5] of the dynamics of forecasts of capacity and nuclear energy production until 2050 showed that the highest growth rates are characteristic of the states of the Far East and Eastern Europe.

The rapid development of new technologies in the electric power industry is facilitated by the proper management of energy systems and the increased use of information and telecommunication technologies. In the electric power industry, digital technologies play a crucial role in monitoring and managing the production, transmission and distribution of electricity.

Growth incentives are provided not only in the "energy" areas of innovation, such as digital energy platforms, intelligent control systems and power electronics, but also in the technology of the "Internet of Things" (digital sensors, sensors, actuators and communications). Digital financial technologies (smart blockchain contracts, decentralized autonomous organizations). For example, the authors in [6] consider the experience of using the Internet of Things (IoT) technology, the technical principles and development goals of IoT for China's electricity system. The authors of [7] provided an overview of hardware-software "hardware" infrastructures (SDHI).

In Russia, as in Europe, China and America, the use of smart grids is a stage in the modernization of energy systems [8,9]. Smart Grid technology is a system that optimizes energy consumption by allowing redistribution of electricity. "Intelligent" networks are a set of technical tools that allows you to quickly change the characteristics of the electrical network. At the technological level, electric networks, consumers and electricity producers are combined into a single automated system, which in real time allows you to monitor and control the operating modes of all participants in the process [10].

One of the types of automated process control systems are SCADA systems [11]. SCADA systems are planning and data collection systems. Software for SCADA-systems can run on computers with various characteristics, in a wide range of parameters of controlled systems $[12,13]$. Given that the information about the systems and protocols of SCADA systems is not protected, work to protect information is quite successful $[14,15]$.

The aim of the work is to develop an intelligent system of experimental control in the energy sector based on the SCADA system. The intelligent experimental control system includes the development and implementation of a modern system designed for operational monitoring and registration of rapidly changing neutron-physical and technological parameters: at the stages of commissioning of a power unit: during physical experiments; during dynamic tests. During the operation of the power unit: during experimental measurements.

\section{Materials and methods}

An intelligent experimental control system designed for a block of nuclear power plants. Physical experiments and tests of the reactor facility equipment during the commissioning of the unit were carried out $[16,17]$. 
During physical experiments, the neutron-physical characteristics of the reactor installation were determined. These characteristics require high accuracy and discreteness of the measured parameters.

Automated process control systems (APCS) used in nuclear power plants allow the collection and recording of analog parameters with a frequency of not more than 1 second, which is not enough for correct calculations when conducting neutron-physical experiments and testing a power unit [18].

There is a need to create a system that allows the reception and registration of analog signals with a frequency of not more than 100 milliseconds. The following principles were used to build a highly accurate registration system:

- Use of modern and technological equipment. ISEC was built using components from German automation equipment manufacturer Beckhoff;

- Using SCADA-system for industrial use and advanced functionality - Indusoft;

- Use of specialized software for data storage - Microsoft SQL Server.

The main functions of intelligent system of experimental control software are: collection and registration of necessary information; preliminary processing of information; archiving and providing protocols with the ability to print; presentation of recorded information in graphical and tabular form; the ability to work with archival data in graphical and tabular form.

Processing and collection of data (technological parameters) is carried out by means of a programmable logic controller (PLC) from the following subsystems: monitoring and control system of the reactor compartment (MCS RC), monitoring and control system of the turbine compartment (MCS TC), the electronic part of the control system (EP CS), neutron flux monitoring equipment (NFME), monitoring, control and diagnostics system (MCDS), electrical equipment control and protection systems (EE CPS), reactimeters, the system of internal reactor control (SIRC), shown in Figure 1.

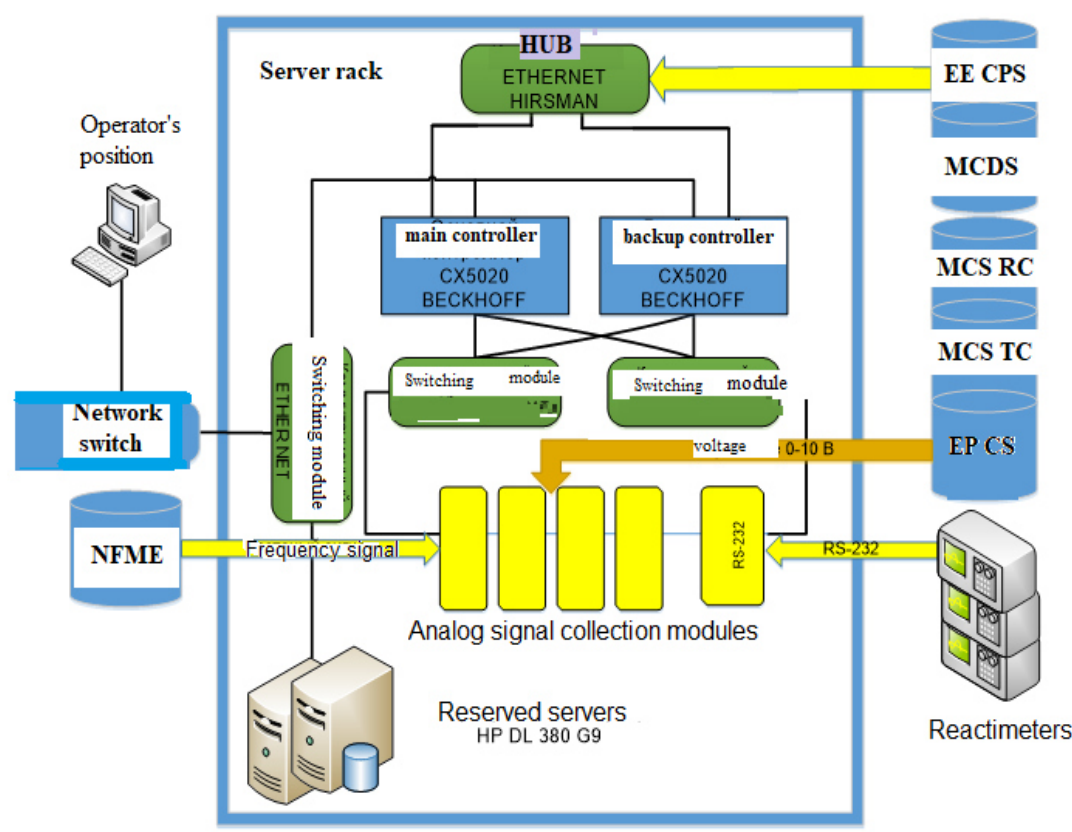

Fig. 1. Block diagram of an intelligent experimental control system. 
The system must meet the following requirements in terms of data collection, processing, storage and presentation: data presentation on at least 20 graphs containing at least 50 changing parameters on each graph; archive depth of at least 2 years; the delay time in data transmission from the PLC to the workstation should not exceed 100 milliseconds.; discretization of writing parameters to the archive should be no more than 100 milliseconds.; the update cycle of discrete and analog data on the screens of a workstation should not exceed 100 milliseconds.; the time of calling the graph on the monitor screen should not exceed 200 milliseconds.

When designing ISEC, the shortcomings identified during the operation of such systems in nuclear, thermal, and wind power plants were taken into account. The software part of the system is often implemented on the basis of SCADA systems Citect, Indusoft, Trace mode [19-21]. These systems are not intended for monitoring and control of fast technological processes; therefore, the following drawbacks arise: limited capabilities of the user interface (working with graphs, presentation of information); there is no possibility of working with archive data in graphical form; with a large number of open trends (charts), there is a delay in updating information on these trends (more than $100 \mathrm{~ms}$ ); unsafe procedure for removing the archive from the ISEC server requires direct access to the database; binding to controllers of a specific manufacturer. This is due to the closeness of the commercial software product and the complexity of its development. In this regard, it was decided to develop its own implementation of software for ISEC, which gives unlimited opportunities to implement customer requirements for the functionality and operator interface, as well as to get away from tight binding to specific hardware components.

The system contains the following equipment: automated workstation of the starter starter; instrument rack reactometers and power supplies ionization chambers; reception and recording parameters of the instrument rack; ionization chambers with junction box.

When designing the server side of the software, the experience of using such systems when starting up power units at nuclear power plants was taken into account to provide for all possible critical points. Most requirements from the personnel of nuclear plants can be formulated as follows:

data recording from subsystems without losses in any operation mode;

reducing the sampling time of the technological data archive;

support for custom formats when uploading data.

To reduce the load when writing data to the database and reading the archive, a microservice architecture was chosen. This solution allows you to divide large tasks into small isolated subtasks and link them by means of the message queue. A pool of data collection services, a pool of services for selecting an archive, a pool of services for working with the client part of the application, and services for internal monitoring of the system were designed.

Data collection services were distributed so that each service supported a specific protocol. At the time the unit was commissioned and tested, the following protocols were implemented: OPCUA, Modbus, EtherCAT, SNMP, NTP. Each service received a given one with different discreteness and assigned a time stamp, in addition, it saved the time stamp of the source, if any. Further, the technological data falls into the message queue. From the message queue, several services are selected for recording data from short-term storage and into the table of recent values. Thanks to such an organization, it is possible to implement any protocol in any language and subsequent integration into the system.

Reducing the selection of data from the entire archive is an mportant issue in the development of an automated process control system. When developing the architecture of 
the database and data warehouse, the specifics of data requests at the nuclear power plant were taken into account. Most often, data is needed for all subsystems and all signals. Another feature is that data sampling with duplication is necessary to confirm the presence of the actual registration of the event. For this, a regulatory database with reference information, databases with current parameter values, a database with the latest data for a floating period ( 1 hour, 2 hours, 5 hours, days) and a long-term data storage were allocated. In a production environment, page-level data compression is used and a grouping of timestamps is separately allocated to reduce storage. But when using and testing, a limited license was used. The figures given in the article were measured without compression and grouping of timestamps.

A data warehouse is a partitioned table divided into partitions depending on the system settings and the number of disks (Figure 2). The hard drive can be located either on a separate RAID-array, or on a RAID-array shared with the database. The storage contains only technological data from all subsystems. The database contains data from directories, magazines, user settings. A table with operational values is located in the server RAM, which minimizes the delay when accessing data. During testing, the version of MSSQL 2012 R2 was used. An important condition in the design of the system was the support of various options for unloading from the archive:

1. Unloading in the format (timestamp1, ... Timestamp N, data, quality, tag name);

2. Unloading in the format (time stamp, name of tag1, quality1, ...., name of tag $\mathrm{N}$, quality N)

It was also possible to specify when requesting the archive not to upload duplicate values, but to upload only the changes. Using the second format of uploading and requesting only changes in values, the volume and time of sampling are significantly reduced. The client application receives data from data access services in real time.

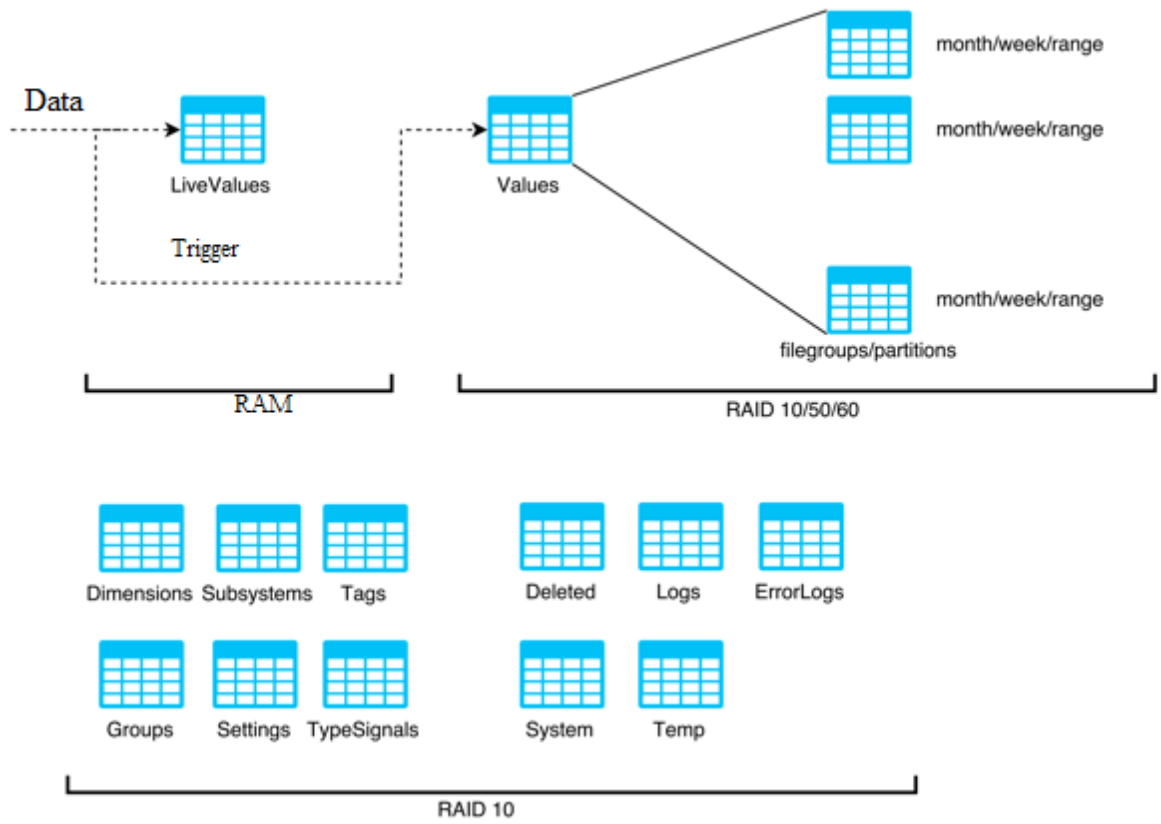

Fig. 2. Organization of data storage. 


\section{Results}

As a result, software was fully implemented for the nuclear power unit that fully complies with the initial technical requirements. Implemented additional functions for the humanmachine interface (Figure 3).
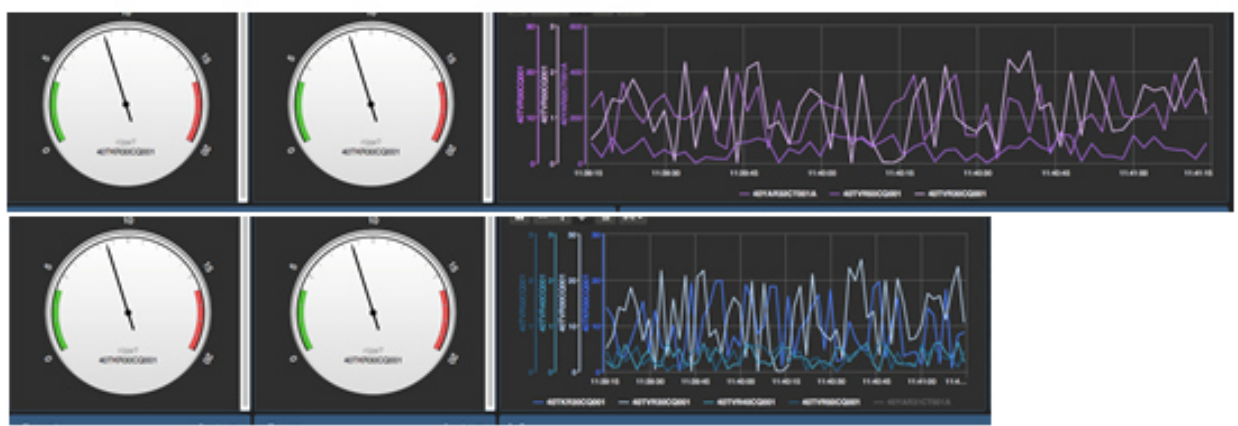

Fig. 3. Operator interface.

The following improvements were made: the ability to use controllers of other manufacturers supporting the OPCUA protocol was implemented; completely redesigned data storage system in terms of database and data storage; a data access service has been developed; allows continuous experiments without time limits without data loss with a guaranteed registration cycle of 100 milliseconds; implemented a flexible, modern humanmachine interface; implemented a software base for further processing and adding new tasks without significant labor costs. Figure 4. presents a graph of the intellectual system of experimental control, which allows you to collect and register analog parameters with a frequency of up to 10 times per second.

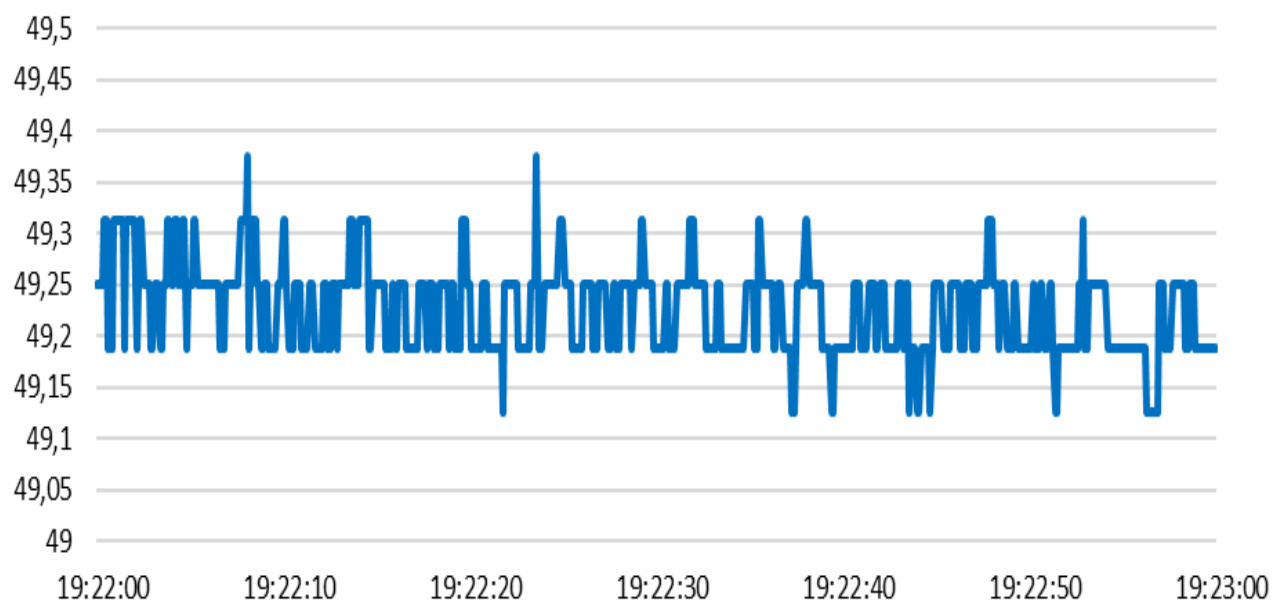

Fig. 4. The neutron flux density in the range of Works-2.

\section{References}

1. O. Popel, Russian J. General Chemistry 6, 95-106 (2008)

2. B. Kroposki, J. Mod. Power Syst. Clean Energy 5, 831-837 (2017) 
3. https://www.irena.org/newsroom/pressreleases/2018/Feb/EU-Doubling-Renewables-by2030-Positive-for-Economy

4. A. Makarova, T. Mitrova, V. Kulagin Forecast of energy development World and Russia 2019 (Moscow: INEI RAS) (2019)

5. Z. Khanalieva, Economic Sciences 166, 157-161 (2018)

6. Y. Wang, Z. Wang, H. Zhao Dand Zhang, Applied Mechanics and Materials 742, 708716 (2015)

7. A. Roozbeh, J. Soares, G. Maguire, F. Wuhib, C. Padala, M. Mahloo, D. Turull, V. Yadhav, D. Kostić, IEEE Communications Surveys \& Tutorials 20 (3), 2454 - 2485 (2018)

8. V. Nesterchuk, A. Pitirimova, D. Fugarov, Science today: challenges and decisions, 5051 (2018)

9. A. Teksler, Energy Policy 5, 3-6 (2018)

10. Annor-Asante, M. Pranggono, Wireless Pers Commun 101, 1357-1377 (2018)

11. V. Krymsky, L. Zhalbekov, R. Imilbaev, A. Yunusov, Electrotechnical and information systems and systems 2, 70-79 (2013)

12. I. Poberezhny, A. Sviridov, Technology and Education 4, 50-53 (2018)

13. P. Trubitcin, Information management and processing systems 1, 94-100 (2017)

14. S. Nazir, M. Kaleem, 3rd Int. Conf. on Emerging Trends in Engineering, Sciences and Technology, 1-4 (2018)

15. A. Lochthofen, D. Sommer, Progress in Nuclear Energy 84, 103-107 (2015)

16. A. Yelshin, D. Zhukovsky, Issues of atomic science and technology. Series: Nuclear and Reactor Constants 1, 17-25 (2018)

17. O. Dreganov, O. Korotkova, DITI Bulletin 1, 40-5 (2017)

18. V. Kishkin, A. Narits, Reports of the Belarusian State University of Informatics and Radioelectronics 2, 13-15 (2015)

19. O. Mnushko, Bulletin of the National Technical University Kharkov Polytechnic Institute. Series: Computer Science and Modeling 24, 117-28 (2018)

20. V. Annenkov, Bulletin of Science and Education 8, 33-36 (2016)

21. I. Kolosok, A. Korkina, A. Tikhonov, Energy systems research 1, 100-107 (2018) 